\title{
Joint Optimization of Spectrum Sensing for Cognitive Radio Networks
}

\author{
Ling Luo, Chittabrata Ghosh, and Sumit Roy \\ Dept. of Electrical Engineering \\ University of Washington \\ Seattle, WA 98195 \\ Email: luol, ghoshc, sroy@u.washington.edu
}

\begin{abstract}
Cognitive radio networks require fast and reliable spectrum sensing to achieve high network utilization by secondary users. Current optimization approaches to spectrum sensing to-date have focussed on maximizing aggregate throughput while considering only a single parameter variable pertinent to sensing, notably the threshold or duration, but not both. In this work, we consider joint minimization of the average detection time for finding a spectrum hole as a function of both parameters. We show that the resulting non-convex problem is actually biconvex under practical conditions and solve for a global optimum detection time. Numerical results show that the proposed approach can considerably improve system performance in terms of the mean time to detect a spectrum hole.
\end{abstract}

\section{INTRODUCTION}

The shift to digital television broadcasting in June 2009 has opened up typical "white spaces" [1] in the VHF and UHF bands. As a result, the Federal Communications Commission (FCC) is encouraging use of these under-used spectrum by unlicensed users [2] to enhance overall utilization. The unlicensed users must be cognitive, i.e., possess the attribute spectrum sensing in order to detect available white spaces and subsequent spectrum sharing. In such a scenario, the TV broadcasting stations and receivers constitute the primary network while the unlicensed users attempting to use white spaces opportunistically are secondary users.

Efficient spectrum sensing is critical in the coexistence of primary and secondary users in licensed bands. Incorrect decisions at the sensing stage leads to two consequences: a) mis-detection of active primary users and imposing inadmissible interference to them or b) missed transmission opportunity when a primary channel is idle. Thus, sensing performance is evaluated in terms of two probabilities: probability of false alarms (primary users present but detected as idle by secondary users) and probability of mis-detection (idle bands being detected as active by secondary users). These are functions of both the sensing threshold as well as the sensing duration.

Liang et. al. [3] proposed an optimal sensing $d u$ ration in order to maximize achievable throughput of the secondary network. Later, the authors extended this single frame optimization approach over multiple slots. Quan et. al. [4] developed an optimization approach to maximize the aggregate throughput for optimal sensing thresholds for each sub-band. However no work to date considers optimization of sensing with respect to both threshold and integration time - this is the main contribution of our work. Increasing the detection threshold increases both the false alarms of primary users and successful detections of spectrum holes. Similarly, integration over longer duration improves spectrum sensing performance for a given threshold of mis-detections.

Accordingly, we

- Formulate an optimization problem of minimizing mean time to detection subject to constraints on the probabilities of missed detection and false alarm;

- Convert the resulting non-convex problem into a bi-convex problem that allows ready computation of the desired optimal values of the key variables (integration time and threshold). time.

\section{System Model}

\section{A. Channel Searching Model}

We consider a scenario where the spectrum is composed of $M$ contiguous channels, each of bandwidth $B_{c}$. We assume that all secondary users are controlled by a central controller to search for available channels. We consider simple one-stage sensing techniques such as random and serial search in this paper. Random search looks for a spectrum hole (available channel) by randomly choosing a channel, while serial search sequentially searches over the entire spectrum, as depicted in Fig.1, until a free channel is found. 


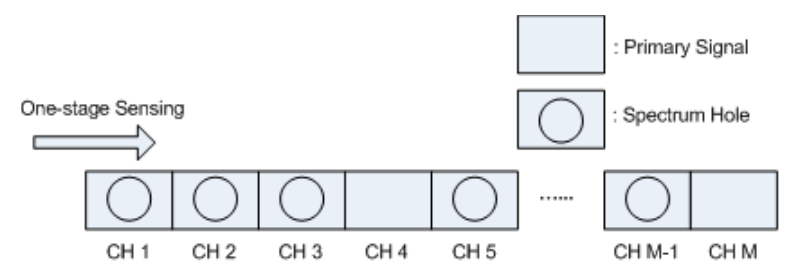

Fig. 1. Channel searching model

We assume that channel state is described by a set of i.i.d Bernoulli variables, and the expected number of available channels is $L(<<M$ typically). Thus the probability of each channel being free is $L / M$. The average number of search steps to detect an available channel for both random search and serial search for small $L / M$ is given by [6] :

$$
\overline{S_{d e t}}=\frac{M}{P_{d} L},
$$

where $P_{d}$ is the detection probability of an available channel.

\section{B. Signal Model}

We consider a binary hypothesis test for spectrum sensing of the $k$-th channel as follows:

$$
\begin{aligned}
& H_{0, k}: \quad R_{k}=N_{k}, \\
& H_{1, k} \quad: \quad R_{k}=H_{k} S_{k}+N_{k},
\end{aligned}
$$

where $R_{k}$ is the received signal at the detector of the secondary user, $S_{k}$ is the primary transmitted signal, and $H_{k}$ is the channel gain between primary transmitter and the secondary receiver. We assume that $N_{k}$ is an i.i.d Gaussian random process with mean zero and variance $E\left[\left|N_{k}\right|^{2}\right]=\sigma_{n}^{2}$. We also assume that $H_{k}$ is an i.i.d random process with mean zero and variance $E\left[\left|H_{k}\right|^{2}\right]=\sigma_{h}^{2}$ and $S_{k}$ is the fixed power of the primary user, which is normalized to 1 .

\section{Energy Detector}

The channel status is sensed based on energy detection with $N$ samples. Thus, the sensing (integration over $N$ samples) duration is $T_{i}=N / B_{c}$, and the test statistic for the energy detector for the $k$-th channel is:

$$
Y_{k}=\frac{1}{N} \sum_{i=1}^{N}\left|R_{k}(i)\right|^{2} .
$$

We denote the probability of successful detection for availability of the $k$-th channel as $P_{d}^{k}$ and the false alarm probability as $P_{f a}^{k}$. Because $H_{k}$ is i.i.d:

$$
\begin{gathered}
P_{d}^{k}=P_{d}, \forall k, \\
P_{f a}^{k}=P_{f a}, \forall k .
\end{gathered}
$$

Here, the detection probability $P_{d}$ is the probability for successfully detection of an available channel under hypothesis $H_{0, k}$. The false alarm probability $P_{f a}$ is the probability of miss-detection of a primary signal under hypothesis $H_{1, k}$.

For large value of $N$, by applying Central Limit Theorem, we approximate $Y_{k}$ to be a Gaussian random variable whose mean and variance under the two hypothesis $\left(H_{0}\right.$ corresponds to channel idle and $H_{1}$ denotes channel busy) are given by:

$$
\begin{aligned}
E\left[Y_{k} \mid H_{0, k}\right] & =\sigma_{n}^{2}, \\
E\left[Y_{k} \mid H_{1, k}\right] & =(\gamma+1) \sigma_{n}^{2}, \\
\operatorname{Var}\left[Y_{k} \mid H_{0, k}\right] & =\frac{2}{N} \sigma_{n}^{4}, \\
\operatorname{Var}\left[Y_{k} \mid H_{1, k}\right] & =\frac{2}{N}(\gamma+1)^{2} \sigma_{n}^{4},
\end{aligned}
$$

where $\gamma=\sigma_{h}^{2} / \sigma_{n}^{2}$ is the per-sample signal-to-noise ratio (SNR).

For a given sensing threshold $x, P_{d}$ and $P_{f a}$ are given by:

$$
\begin{aligned}
P_{d}(x, N) & =\operatorname{Pr}\left(Y_{k}<x \mid H_{0, k}\right) \\
& =1-Q\left(\frac{x-\sigma_{n}^{2}}{\sigma_{n}^{2} \sqrt{2}} \sqrt{N}\right), \\
P_{f a}(x, N) & =\operatorname{Pr}\left(Y_{k}<x \mid H_{1, k}\right) \\
& =1-Q\left(\frac{x-(\gamma+1) \sigma_{n}^{2}}{\sigma_{n}^{2}(\gamma+1) \sqrt{2}} \sqrt{N}\right),
\end{aligned}
$$

where the Q-function is:

$$
Q(x)=\frac{1}{2 \pi} \int_{x}^{\infty} e^{t^{2} / 2} d t .
$$

\section{Optimization of Average Detection Time}

Based on the detection and false alarm probabilities derived in the previous section, we jointly optimize two fundamental parameters of the energy detector, i.e., the sensing threshold and the integration time, to minimize the mean time to detect an available channel.

\section{A. Detection Time}

We denote the channel switching time and integration time in the energy detector as $T_{s}$ and $T_{i}$, respectively. $T_{s}$ is determined by the phase locked loop (PLL) design in the receiver circuitry and is also known as the PLL settling time [5]. On the other hand, $T_{i}$ in the energy 
detector is only determined by the width of sensing bandwidth and the number of sensing samples. Thus, the mean time to detect an available channel is:

$$
\begin{aligned}
\overline{T_{\text {det }}} & =\overline{S_{d e t}}\left(T_{s}+T_{i}\right), \\
& =\frac{M}{P_{d} L}\left(T_{s}+\frac{N}{B_{c}}\right) .
\end{aligned}
$$

\section{B. Problem Formulation}

In this work, we seek to minimize the average detection time of an available channel for secondary users. A higher average detection time leads to longer delay to allocate a feasible channel for an incoming secondary user, hence decreasing their throughput. Moreover, if a primary user becomes active in a channel, being used a secondary user, switching (to another available channel) must be accomplished by a secondary user within a short delay for session continuity.

Considering Eq. (7), we note a trade-off between the average number of detection steps and the integration time: a longer integration time leads to a better $P_{d}-P_{f a}$ performance, hence reducing the mean number of detection steps, and vice versa. As is shown in Section II-C, the integration time is proportional to the number of sensing samples. Moreover, for any given integration time, the energy detector with higher sensing threshold results in higher detection and false alarm probabilities. Thereby, this paper focuses on jointly optimizing the sensing threshold and the number of sensing samples in order to minimize the average detection time.

Therefore, the joint optimization problem is mathematically formulated as below:

$$
\begin{aligned}
\min _{x, N} & \overline{T_{\text {det }}}=\frac{M}{P_{d} L}\left(T_{s}+\frac{N}{B_{c}}\right) \\
\text { subject to } & \text { 1) } P_{d}(x, N) \geq \alpha ; \\
& 2) P_{f a}(x, N) \leq \beta
\end{aligned}
$$

where $P_{d}(\cdot, \cdot)$ and $P_{f a}(\cdot, \cdot)$ are detection and false alarm probabilities, respectively and are functions of sensing threshold and the number of sensing samples. The parameters $\alpha$ and $\beta$ denote appropriate constraints on detection and false alarm probabilities.

\section{Optimization of Average Detection Time}

The formulation in Eq. 8 is, in general, a non-convex optimization problem. For practical systems, a high detection probability $\alpha$ and a low false alarm probability $\beta$ are desired. In this sub-section, we will prove that the original non-convex problem can be transformed into a biconvex optimization problem [7] when $(\alpha>0.5)$ and $(\beta<0.5)$. Biconvex problems are in general global optimization problems, unlike convex optimization problems. Hence, we resort to transform our optimization problem into a problem on biconvex optimization.

Definition: A function $f: B \rightarrow \mathbb{R}$ on a biconvex set $B \subset X \times Y$ is called a biconvex function [7] on $B$ if:

$$
f_{x}(.):=f(x, .): B_{x} \rightarrow \mathbb{R}
$$

is a convex function on $B_{x}$ for every fixed $x \in X$ and

$$
f_{y}(.):=f(., y): B_{y} \rightarrow \mathbb{R}
$$

is a convex function on $B_{y}$ for every fixed $y \in Y$.

An optimization problem of the form:

$$
\min \{f(x, y):(x, y) \in B\}
$$

is said to be a biconvex optimization problem if the feasible set $B$ is convex on $X \times Y$ and the objective function $f$ is biconvex on $B$.

To prove the above optimization problem to be biconvex, we need to prove that each of the constraints satisfy the convexity property. We introduce variables $\varepsilon=\frac{x-\sigma_{n}^{2}}{\sigma_{n}^{2} \sqrt{2}}$, $\eta=\sqrt{N}$ and let $\tau=\frac{T_{s}}{B_{c}}$. We denote $\mathbb{E}$ and $\mathbb{H}$ as the feasible sets for $\varepsilon$ and $\eta$. Combining Eqs. (5) and (6), the original optimization problem in Eq. (8) is re-written as:

$$
\begin{array}{cl}
\min _{\varepsilon, \eta} & \overline{T_{\text {det }}}(\varepsilon, \eta)=\frac{M}{B_{c} L} \frac{\tau+\eta^{2}}{1-Q(\varepsilon \eta)} \\
\text { subject to } & 1) Q(\varepsilon \eta) \leq 1-\alpha ; \\
& 2)-Q\left(\frac{\sqrt{2} \varepsilon-\gamma}{\sqrt{2}(\gamma+1)} \eta\right) \leq \beta-1 ; \\
& 3) \eta \geq 1
\end{array}
$$

Lemma 1: Given $\varepsilon=\tilde{\varepsilon} \in \mathbb{E}$, the function $Q(\tilde{\varepsilon} \eta)$ is convex in $\eta$ if $P_{d}>1 / 2$.

Proof: Taking the second partial derivative of $Q(\tilde{\varepsilon} \eta)$ with respect to $\eta$ gives:

$$
\frac{\partial^{2} Q(\hat{\varepsilon} \eta)}{\partial \eta^{2}}=\frac{\tilde{\varepsilon}^{3} \eta}{\sqrt{2 \pi}} e^{-(\tilde{\varepsilon} \eta)^{2} / 2} .
$$

Since $Q(\cdot)$ is a monotonic decreasing function and $Q(0)=1 / 2$, the condition that $P_{d}=1-Q(\tilde{\varepsilon} \eta)>1 / 2$ is equivalent to the condition that $\tilde{\varepsilon} \eta>0$. Because $\eta>0$, we have $\tilde{\varepsilon}>0$, and this condition satisfies the positivity of $\frac{\partial^{2} Q(\tilde{\varepsilon} \eta)}{\partial \eta^{2}}$. Thereby $Q(\tilde{\varepsilon} \eta)$ is convex in $\eta$.

Lemma 2: Given $\varepsilon=\tilde{\varepsilon} \in \mathbb{E}$, the function $-Q\left(\frac{\sqrt{2} \tilde{\varepsilon}-\gamma}{\sqrt{2}(\gamma+1)} \eta\right)$ is convex in $\eta$ if $P_{f a}<1 / 2$.

Proof: Taking the second partial derivative of the 
function with respect to $\eta$ gives:

$$
\begin{aligned}
& \frac{\partial^{2}}{\partial \eta^{2}}\left[-Q\left(\frac{\sqrt{2} \tilde{\varepsilon}-\gamma}{\sqrt{2}(\gamma+1)} \eta\right)\right] \\
= & -\frac{\eta}{\sqrt{2 \pi}}\left(\frac{\sqrt{2} \tilde{\varepsilon}-\gamma}{\sqrt{2}(\gamma+1)}\right)^{3} e^{-(\sqrt{2} \tilde{\varepsilon}-\gamma)^{2} \eta^{2} /\left[4(\gamma+1)^{2}\right]}
\end{aligned}
$$

Similarly, because $P_{f a}=1-Q\left(\frac{\sqrt{2} \varepsilon-\gamma}{\sqrt{2}(\gamma+1)} \eta\right)<1 / 2$, we have $\frac{\sqrt{2} \tilde{\varepsilon}-\gamma}{\sqrt{2}(\gamma+1)}<0$. The positivity of the second partial derivative proves the convexity of $-Q\left(\frac{\sqrt{2} \varepsilon-\gamma}{\sqrt{2}(\gamma+1)} \eta\right)$ in $\eta$.

Lemma 3: Given $\varepsilon=\tilde{\varepsilon} \in \mathbb{E}$, the function $\overline{T_{\text {det }}}(\tilde{\varepsilon}, \eta)$ is convex in $\eta$, if $P_{d}>1 / 2$.

Proof: Taking the second partial derivative of the function with respect to $\eta$ gives:

$\frac{\partial^{2} \overline{T_{d e t}}(\tilde{\varepsilon}, \eta)}{\partial \eta^{2}}=\frac{\partial^{2}}{\partial \eta^{2}} \frac{M \tau}{B_{c} L} F(\tilde{\varepsilon}, \eta)^{-1}+\frac{\partial^{2}}{\partial \eta^{2}} \frac{M}{B_{c} L} \frac{\eta^{2}}{F(\tilde{\varepsilon}, \eta)}$,

where $F(\varepsilon, \eta)=1-Q(\varepsilon, \eta)>0$.

Let $G(\varepsilon, \eta)=\frac{\partial F(\varepsilon, \eta)}{\partial \eta}=\frac{\varepsilon}{\sqrt{2 \pi}} e^{-\varepsilon^{2} \eta^{2} / 2}$, and thus

$$
\begin{aligned}
& \frac{\partial^{2}}{\partial \eta^{2}} \frac{M \tau}{B_{c} L} F(\tilde{\varepsilon}, \eta)^{-1} \\
= & \frac{\partial}{\partial \eta} \frac{-M \tau}{B_{c} L} F(\tilde{\varepsilon}, \eta)^{-2} G(\tilde{\varepsilon}, \eta) \\
= & \frac{M \tau}{B_{c} L} F(\tilde{\varepsilon}, \eta)^{-3} G(\tilde{\varepsilon}, \eta)[2 G(\tilde{\varepsilon}, \eta) \\
& \left.+F(\tilde{\varepsilon}, \eta) \tilde{\varepsilon}^{2} \eta\right] \\
& \frac{\partial^{2}}{\partial \eta^{2}} \frac{M}{B_{c} L} \frac{\eta^{2}}{F(\tilde{\varepsilon}, \eta)} \\
= & \frac{\partial}{\partial \eta} \frac{M}{B_{c} L} \frac{2 \eta F(\tilde{\varepsilon}, \eta)-\eta^{2} G(\tilde{\varepsilon}, \eta)}{F(\tilde{\varepsilon}, \eta)^{2}} \\
= & \frac{2 M}{B_{c} L} \frac{[F(\tilde{\varepsilon}, \eta)-\eta G(\tilde{\varepsilon}, \eta)]^{2}}{F(\tilde{\varepsilon}, \eta)^{4}}
\end{aligned}
$$

As we have shown in Lemma 1 that $\tilde{\varepsilon}>0$, hence $G(\tilde{\varepsilon}, \eta)>0$. Because $M, \tau, B_{c}$ and $L$ are of positive values, (15) is obviously positive and (16) is nonnegative, leading to the positivity of (14). Thus $\overline{T_{\text {det }}}(\tilde{\varepsilon}, \eta)$ is convex in $\eta$.

Lemma 4: Given $\varepsilon=\tilde{\varepsilon} \in \mathbb{E}$, the optimization problem (12) is convex in $\eta$, if $P_{d}>1 / 2$ and $P_{f a}<1 / 2$.

Proof: Follows upon combining Lemmas 1-3.

Lemma 5: Given $\eta=\tilde{\eta} \in \mathbb{H}$, the optimization problem (12) is convex in $\varepsilon$, if $P_{d}>1 / 2$ and $P_{f a}<1 / 2$.

Proof: This can be proved using a similar technique by showing that $Q(\varepsilon \tilde{\eta}),-Q\left(\frac{\sqrt{2} \varepsilon-\gamma}{\sqrt{2}(\gamma+1)} \tilde{\eta}\right)$ and $\overline{T_{\text {det }}}(\varepsilon, \tilde{\eta})$

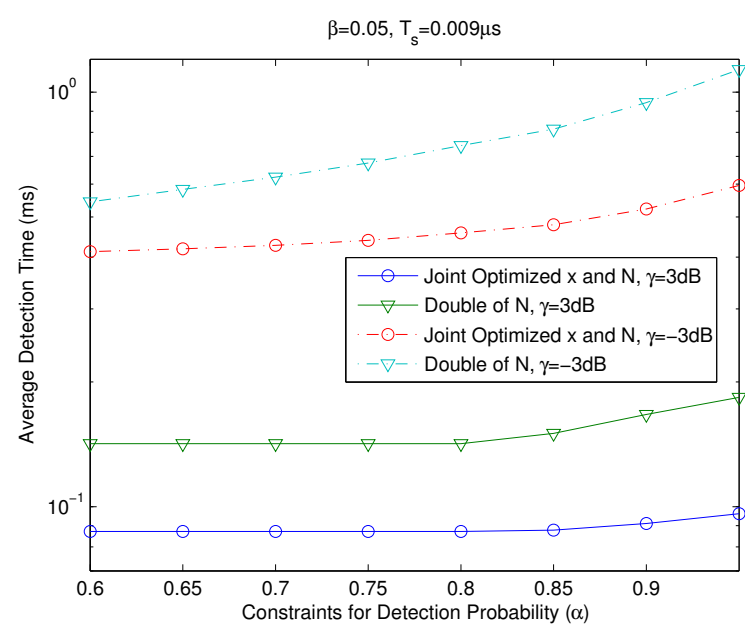

Fig. 2. Minimized average detection time: varying $\alpha$

are all convex in $\varepsilon$ under the constraints $P_{d}>1 / 2$ and $P_{f a}<1 / 2$.

Theorem 1: The optimization problem (12) is biconvex for $\varepsilon \in \mathbb{E}$ and $\eta \in \mathbb{H}$, if $P_{d}>1 / 2$ and $P_{f a}<1 / 2$.

Proof: Because $\varepsilon$ and $\eta$ are independent, this can be proved from Lemma 4 and Lemma 5.

\section{Finding Optimal Points: Numerical Results}

Iterative algorithms for finding the global optimum points in the biconvex optimization problems are discussed in [7]. Here we use GOP algorithm of [8] to determine the solution for our biconvex optimization problem.

We present results for two scenarios: for fixed $(\beta)$ and varying $(\alpha)$, and vice versa. We let $M=100, L=4$ and $B_{c}=6 \mathrm{MHz}$ conforming to the bandwidth of a TV channel.

We note from the equivalent problem (12) that both $\tau$ (proportional to $T_{s}$ ) and $\gamma(\mathrm{SNR})$ play important roles in determining the minimum average detection time. Thus, we consider two SNR cases $(\gamma= \pm 3 d B)$ and also two values of switching time $T_{s}=0.005 \mu s$ (type I PLL) and $T_{s}=20 \mu s$ (type II PLL).

Fig. 2 shows the results for type I circuit, where the circuit switching time is much smaller than the integration time. The average detection time increases with $\alpha$ as expected. We also note that the minimum average detection time is approximately constant for $0.6 \leq P_{d} \leq 0.85$. This is because the local optimal point $\varepsilon_{\text {optimal }}=\frac{Q^{-1}(1-\beta)(\gamma+1)}{\eta_{\text {optimal }}}+\frac{\gamma}{\sqrt{2}}$ is invariant w.r.t $\alpha$ while satisfying the constraint $Q\left(\varepsilon_{\text {optimal }} \eta_{\text {optimal }}\right) \leq 1-\alpha$. Thereafter, the optimal mean detection time is strictly increasing for $P_{d}>0.85$, because $\varepsilon_{\text {optimal }}=\frac{Q^{-1}(1-\alpha)}{\eta_{\text {optimal }}}$. 


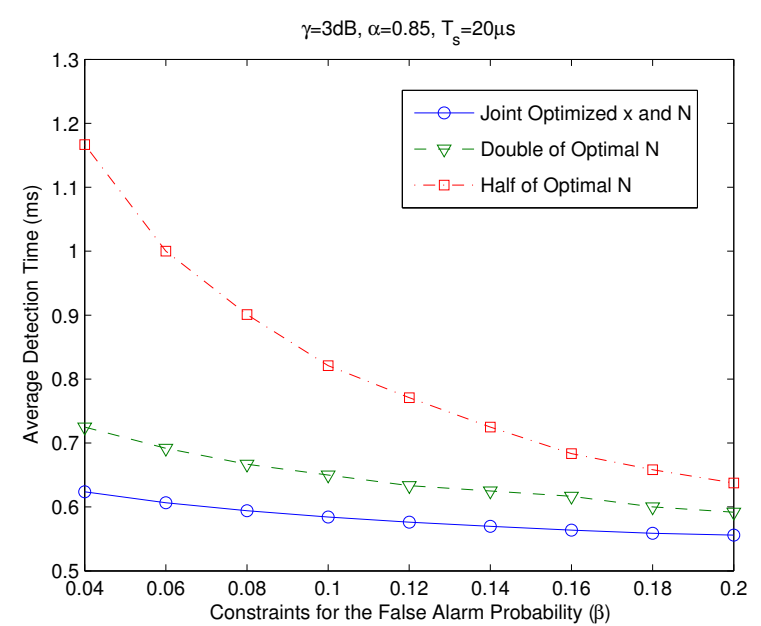

Fig. 3. Minimized average detection time: varying $\beta, \mathrm{SNR}=3 \mathrm{~dB}$

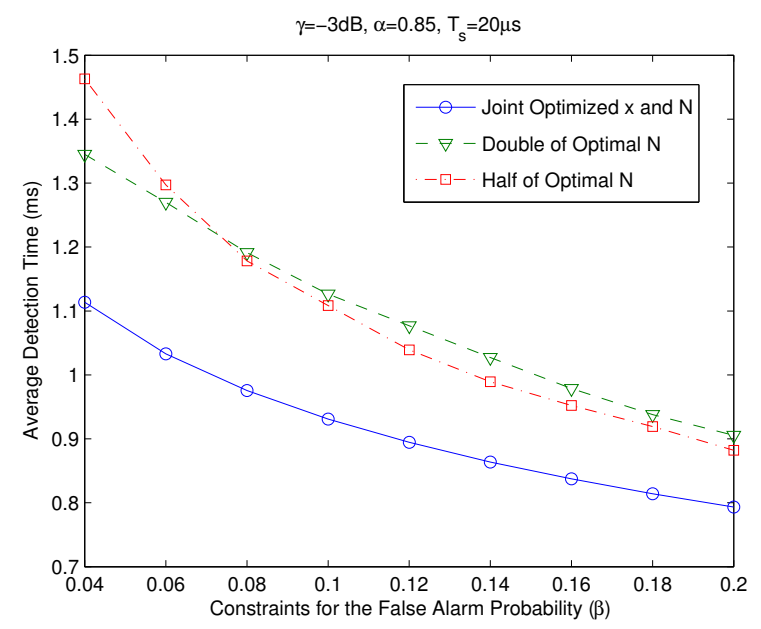

Fig. 4. Minimized average detection time: varying $\beta, \mathrm{SNR}=-3 \mathrm{~dB}$

Figs. 3-4 shows the results for type II circuit, where the circuit switching time is close to the integration time. It can be also noticed that all curves are going down as $\alpha$ increases, because the higher constraints for false alarm probability leads to less integration time, hence decreasing the minimum average detection time. It can be also observed that the optimal values of detection time are at least $20 \%$ lower than taking half or double of the optimal number of sensing samples.

\section{CONCLUSION}

This paper proposes a biconvex optimization approach for spectrum sensing in cognitive radio networks. We formulate a joint optimization problem regarding both the sensing threshold and the number of sensing samples in the energy detector, to minimize the average detection time of an available channel. Although this problem in, we prove that it can be transformed into a biconvex problem under practical conditions. Numerical results show that the proposed optimal approach can significantly decrease the mean time to detect a spectrum hole.

\section{REFERENCES}

[1] M. Nekovee, "Quantifying the Availability of TV White Spaces for Cognitive Radio Operation in the UK,' ICC'09, pp. 1-5, June 2009.

[2] J. Mitola III, Cognitive radio: An Integrated Agent Architecture for Software Defined Radio. Ph.D. Thesis, Swedish Royal Institute of Technology, 2000.

[3] Y-C. Liang, Y. Zeng, E. C. Y. Peh, and A.T. Hoang, "Sensingthroughput tradeoff for cognitive radio networks," IEEE Trans. on Wireless Commun., vol. 7, no. 4, pp. 1326-1337, Apr. 2008.

[4] Z. Quan, S. Cui, A. H. Sayed, and H. V. Poor, "Optimal multiband joint detection for spectrum sensing in cognitive radio networks," IEEE Transactions on Signal Processing, vol. 57, no. 3, pp. 1128-1140, Mar. 2009.

[5] L. Luo, N. M. Neihart, S. Roy, and D. J Allstot, "A Two-stage sensing technique for dynamic spectrum access," IEEE Trans. on Wireless Commun., Vol. 8, No. 6, pp. 3028-3037, June 2009.

[6] L. Luo and S. Roy, "Analysis of search schemes in cognitive radio," SECON'07, pp. 647-654, June 2007.

[7] J. Gorski, F. Pfeuffer, and K. Klamroth, "Biconvex sets and optimization with biconvex functions: A survey and extensions," Math. Meth. of Oper. Res., Vol. 66, No. 3, pp. 373-407, Dec. 2007.

[8] C.A. Floudas and V.Visweswaran, "A global optimization algorithm (GOP) for certain classes of nonconvex NLPs: I. Theory," Computer Chem. Eng., Vol. 14, No. 12, July 1990. 